\title{
Biological reactivity of nanoparticles: mosaics from optical microscopy videos of giant lipid vesicles
}

\author{
Jernej Zupanc, ${ }^{a}$ Andrej Dobnikar, ${ }^{a}$ Damjana Drobne,,${ }^{b, d, e}$ Janez Valant, ${ }^{\mathrm{b}}$ Deniz Erdogmus, ${ }^{\mathrm{c}}$ and Erhan Bas ${ }^{\mathrm{c}}$ \\ a University of Ljubljana, Faculty of Computer and Information Science, Trzaska 25, SI-1000, Ljubljana, Slovenia \\ ${ }^{b}$ University of Ljubljana, Biotechnical Faculty, Department of Biology, Jamnikarjeva 101, SI-1000, Ljubljana, Slovenia \\ ${ }^{\mathrm{C} N o r t h e a s t e r n ~ U n i v e r s i t y, ~ E l e c t r i c a l ~ a n d ~ C o m p u t e r ~ E n g i n e e r i n g ~ D e p a r t m e n t, ~} 365$ Huntington Avenue, Boston, \\ Massachusetts 02115 \\ dozef Stefan Institute, Centre of Excellence in Advanced Materials and Technologies for the Future (CO NAMASTE), \\ Jamova 39, SI-1000 Ljubljana, Slovenia \\ eJozef Stefan Institute, Centre of Excellence in Nanoscience and Nanotechnology (CO Nanocenter), Jamova 39, \\ SI-1000 Ljubljana, Slovenia
}

\begin{abstract}
Emerging fields such as nanomedicine and nanotoxicology, demand new information on the effects of nanoparticles on biological membranes and lipid vesicles are suitable as an experimental model for bio-nano interaction studies. This paper describes image processing algorithms which stitch video sequences into mosaics and recording the shapes of thousands of lipid vesicles, which were used to assess the effect of $\mathrm{CoFe}_{2} \mathrm{O}_{4}$ nanoparticles on the population of 1-palmitoyl-2-oleoyl-sn-glycero-3-phosphatidylcholine lipid vesicles. The applicability of this methodology for assessing the potential of engineered nanoparticles to affect morphological properties of lipid membranes is discussed. @ 2011 Society of Photo-Optical Instrumentation Engineers (SPIE). [DOI: 10.1117/1.3533319]
\end{abstract}

Keywords: nanotoxicology; computational microscopy; video microscopy; giant lipid vesicles; mosaic; image processing; nanoparticles. Paper 10453R received Aug. 12, 2010; revised manuscript received Dec. 2, 2010; accepted for publication Dec. 7, 2010; published online Feb. 8, 2011.

\section{Introduction}

Lipid vesicles are highly adaptive structures with a rich diversity of shapes. Even minute asymmetries in the lipid bi-layers can cause high spontaneous curvatures and deformations of vesicle, causing its shape to range from spherical to pear-shaped, cupshaped, budded, and pearled. ${ }^{1,2}$

Most experimental evidence on membrane behavior is provided by giant lipid vesicles ${ }^{1,3,4}$ which can be formed in various sizes as uni- or multilamellar constructions and are objects of research in diverse areas concerned with lipid membranes. Due to their size, which is on the same order of magnitude as that of cells, they are surrogates for cell membranes and can be observed with a light microscope. Research on lipid vesicles is extensively focused on their conformational behavior and considers preferred shapes, shape transformations, and shape fluctuations. ${ }^{1,5-8}$ Changes and fluctuations in the shape of vesicles has been widely investigated by various techniques, most commonly optical microscopy. ${ }^{7,9-11}$ The preponderance of the published research concerns the observation of individual vesicles or a well-defined group of vesicles.

Recently, research related to biological membranes has been gaining importance due to the products emerging from new technologies. These include drugs and diagnostic tools, as well as ingredients in food and cosmetics, whose primary reaction, at the nanoscale level, is with cell membranes. These products have many beneficial effects but may also provoke a toxic response. ${ }^{12}$ Interactions of nanoparticles with lipid vesicles that have been studied thus far, reveal that nanoparticles induce lipid surface

Address all correspondence to Jernej Zupanc, University of Ljubljana, Faculty of Computer and Information Science, Trzaska 25, SI-1000, Ljubljana, Slovenia. E-mail: jernej.zupanc@fri.uni-lj.si. reconstruction, ${ }^{13}$ physical disruption of lipid membranes, ${ }^{14,15}$ and shape transformations of lipid vesicles. ${ }^{16,17}$ In our previous work ${ }^{17}$ we presented a controllable experimental setup for data acquisition for the purpose of nanoparticle-lipid vesicle interaction studies. We employed light microscopy and recorded multiple micrographs of exposed and unexposed vesicle populations. Vesicles in the micrographs were segmented in order to gather data on their shapes and sizes. The gathered data showed that the vesicle population based approach is suited for this type of research. However, in order to gather more reliable results, a greater quantity of vesicles should be recorded in each population, which is feasible by employing microscopy videos instead of micrographs.

In the work presented in this paper, we introduce a methodology in which changes in shape of lipid vesicles in a population of vesicles are recorded and statistically evaluated. In this experiment, lipid vesicles of 1-palmitoyl-2-oleoyl-sn-glycero3-phosphatidylcholine (POPC) were incubated with $\mathrm{CoFe}_{2} \mathrm{O}_{4}$ nanoparticles. These nanoparticles are often used in biomedicine as an in vivo magnetic nanoparticle hyperthermia-inducing agent. ${ }^{18}$ In addition, the $\mathrm{CoFe}_{2} \mathrm{O}_{4}$ nanoparticles are expected to have the potential to non-specifically react with lipid membranes. It is known that biological potential of nanoparticles is related to their surface chemistry, and accordingly, we tested two types of nanoparticles; uncoated cobalt-ferrite nanoparticles (CF) and negative citrate-coated cobalt-ferrite (CF-CA) nanoparticles.

In order to investigate the morphological characteristics of lipid vesicles in ambient populations, we recorded a video sequence covering a larger area of the sample and containing thousands of lipid vesicles, instead of individual micrographs of

1083-3668/2011/16(2)/026003/10/\$25.00 @ 2011 SPIE 
vesicles as it has typically been performed in such studies. ${ }^{17}$ To allow manual or automatic segmentation of vesicles, the video sequences were transformed into mosaics.

A mosaic, or panorama, is a large format image that has been built by seamlessly stitching numerous smaller images that together cover an area wider than that which can be acquired by a single field of view. Mosaics have been gaining popularity not only among photographers, but also among scientists in various areas, because such approaches can broaden the utility of existing and available equipment. Recently, mosaics have been applied to images produced by microscopy. ${ }^{19-22}$ When hundreds of images must be stitched together to produce a mosaic, manual imaging becomes increasingly difficult and to assure seamless transitions between images, many conditions such as overlap between images, lighting and focus conditions, and viewpoint must be satisfied. These are not easily reconciled and various automated systems have been proposed ${ }^{23,24}$ including commercial mosaicing software (Virtual Slice by MicroBrightField Inc., Williston, Vermont). Although such acquisition of data finds various applications in biology, medicine, and other fields where microscopy mosaics are necessary, it is less appropriate for the observation of more dynamic specimens in a strictly constrained time window.

The population of giant lipid vesicles employed in our experiments is an example of such specimen. The data must be acquired in a short time in order to observe a large area, but all the information required to stitch images into a mosaic, must be retained. Microscopy video sequences as a basis for mosaic stitching, have the potential to resolve both issues and although its applications in biomedical imaging are rare, it is our preferred choice. $^{25}$

The aim of our work was to develop a methodology to enable extraction of information on size and shape transformations of vesicles in lipid vesicle populations incubated in a suspension of nanoparticles. Microscopy video sequences of POPC lipid vesicles incubated in a suspension of non-coated and the negative citrate-coated cobalt-ferrite nanoparticles, were recorded and each video sequence of an investigated area was assembled into a mosaic. Next, the giant lipid vesicles were segmented from the mosaics, and their sizes and shapes were evaluated. Issues of redundant data, computational efficiency, image features enabling selection of high quality frames in terms of image sharpness, stitching process, segmentation of vesicles, and analysis of their morphological characteristics, are discussed and the advantages of a population-based approach to assess dynamic conformational changes of vesicles are considered.

\section{Materials and Methods}

\subsection{Proposed Methodology}

The proposed methodology consists of five steps, as presented in Fig. 1. The first step is the lipid vesicle experiment and the recording of microscopy video sequences of lipid vesicles exposed in different media. The procedure ends with a statistical analysis of the vesicles' shapes in the observed lipid vesicle populations.

\subsubsection{Experimental set up}

Preparation of vesicles and nanoparticles. Giant unilamelar phospholipid vesicles were prepared from 1-palmitoyl-2-oleoyl-

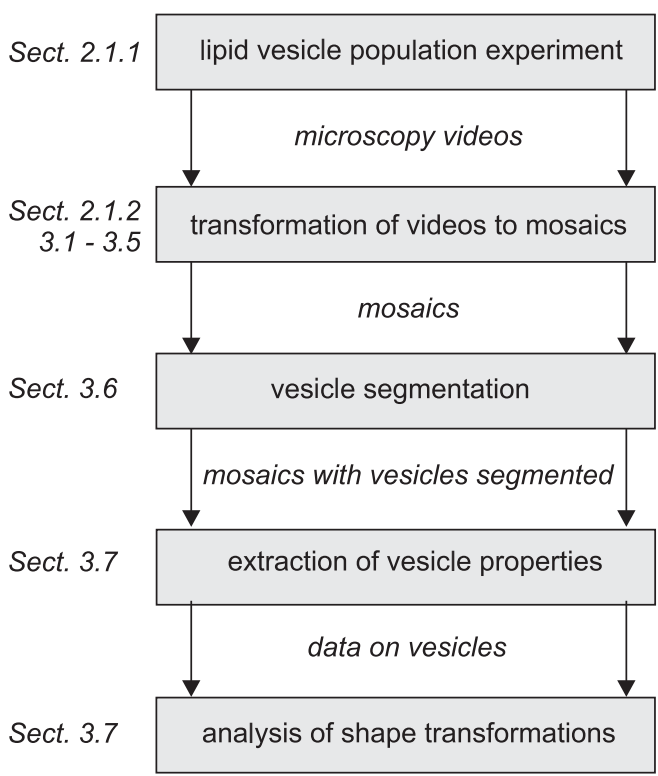

Fig. 1 An outline of the proposed methodology. Shaded boxes present the steps in the methodology and the text in italics gives the outputs of these steps. Starting with the lipid vesicles experiment, we acquire the microscopy videos. Next, these are transformed into mosaics with the algorithms described here. In the next step, a human expert segments vesicles in the mosaics with specific color for each morphological type of vesicle. Data on the properties of these colored vesicles are analyzed to extract underlying knowledge about the vesicle population. The text in italic on the left of the shaded boxes, points to the sections of this paper where the associated step is described in detail.

$s n$-glycero-3-phosphatidylcholine (POPC) at room temperature by the modified electroformation method ${ }^{26}$ as described in detail elsewhere. ${ }^{27}$ They were created in saccharose solution and rinsed with an equi-osmolar glucose solution. The intact membrane is impermeable to sugar molecules, and the composition inside the vesicles, primarily saccharose, differs from the saccharose/glucose composition outside. Because of this, the lipid vesicles' interior appears darker from the surrounding medium in the micrographs and is easier to distinguish from the

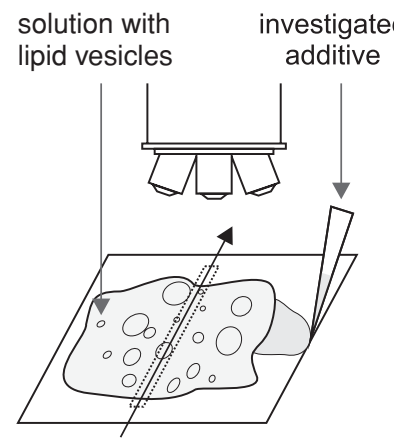

a

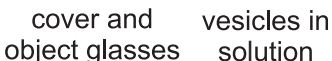
object glasses solution

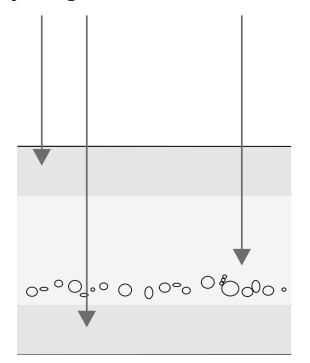

b
Fig. 2 (a) The solution with lipid vesicles on the object glass is covered with a glass plate and the suspension with the investigated additive is added. The place where the videos are recorded is shown and the arrow shows direction of recording. (b) Transverse section of the object and cover glasses and the suspension with lipid vesicles. A majority of the vesicles are in the same focal plane at the bottom of the chamber. The scheme is not to scale. 
background. A 5\% glucose suspension containing lipid vesicles (45 $\mu$ l) was administered to each object glass [Fig. 2(a)] and a strip of silicone gel was applied on two opposite sides of the cover glass to act as a spacer between the cover and the object glass. Subsequently, a droplet of a suspension with investigated additive was added to the vesicles and finally, the additional two edges of the cover glass were filled with the silicon paste to minimize evaporation.

The nanoparticles were synthesized by co-precipitation using $\mathrm{NaOH}$ from aqueous solutions of $\mathrm{Co}$ (II) and $\mathrm{Fe}$ (III) ions at elevated temperatures. The samples of CF were thoroughly washed with water and suspended in an aqueous solution of glucose. The nanoparticles in suspension agglomerate strongly and such agglomeration must be prevented in order to prepare stable suspensions of the nanoparticles. To achieve this, citric acid was adsorbed to the surface of the nanoparticles. The nanoparticles have relatively broad size distribution ranging from 5 to $>15 \mathrm{~nm}$. The smaller nanoparticles are globular, while the larger are octahedral in shape. Energy dispersive x-ray spectroscopy (EDS) showed their stoichiometric composition to be $\mathrm{CoFe}_{2} \mathrm{O}_{4}$. The effects of both non-coated cobalt-ferrite nanoparticles (CF) and the negative citrate-coated cobalt-ferrite nanoparticles (CF-CA) were investigated.

Incubation of vesicles with nanoparticles. Preliminary experiments showed that the small negative buoyancy of the vesicles causes them to collect at the bottom of the suspension. ${ }^{17,28} \mathrm{We}$ have experimentally confirmed that after up to $5 \mathrm{~min}$, a majority of the vesicles are in the same plane, collected near the lower object glass. This allows us to record the vesicles with only minor adjustments to the focal plane. The vesicles were incubated for up to $90 \mathrm{~min}$.

Recording of vesicles. With the specimen mounted to the stage of the light microscope, 1-dimensional video tracks of specimen were recorded [Fig. 2(a)-2(b)] at 400 $\times$ magnification. The width of each track was $195 \mu \mathrm{m}$ and the length, $1 \mathrm{~cm}$. By recording the tracks we captured a subsample of the population where all vesicles of a single track were at approximately the same distance from the place where the nanoparticles had been added.

\subsubsection{Video to mosaic steps}

Each video sequence of approximately 5 min of a selected track on the object glass was then transformed into a mosaic of the entire recorded area. This process consists of multiple steps related to the transformation of videos to mosaics, mosaicing, (Fig. 3) and are described in detail in the Results section of this paper. The output of mosaicing is a single mosaic, stitched together from the selected frames of the video sequence. Some steps of the mosaicing were necessary only for the first video sequence, which involves the training of classifiers for frame quantity reduction and calculation of lens noise. The models (classifiers, measures) generated in these steps can subsequently be used on all remaining video sequences of the experiment. Here, we refer to this video sequence of a single track used in training as the pilot video. It was selected randomly among the videos recorded in the experiment.

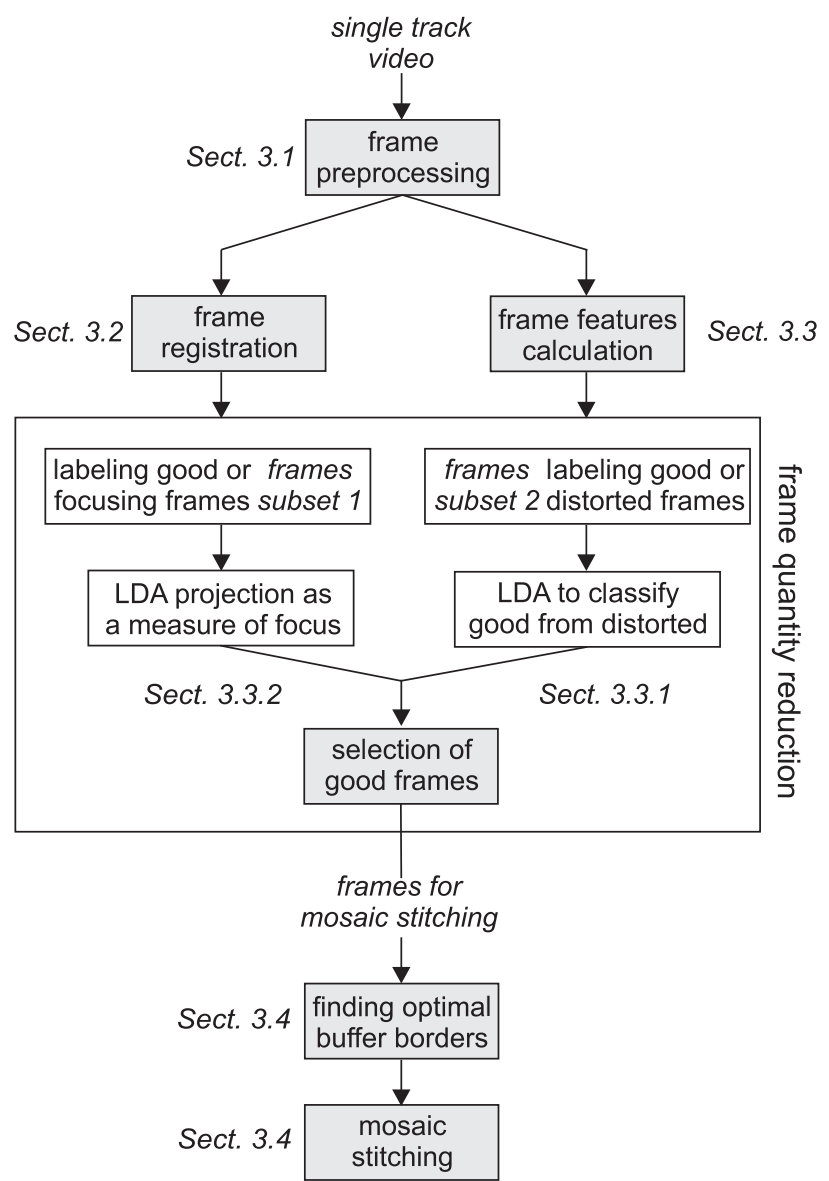

Fig. 3 An outline of the steps required for transforming video sequences to mosaics. Boxes represent processing steps and the text in italics their outputs. Only the first (pilot) video sequence of the experiment is used for training classifiers in the non-shaded steps while the shaded steps are required for all videos. The numbers at the side of each step indicate the section of this paper describing the step in detail. LDA-Linear discriminant analysis.

\subsubsection{Vesicle segmentation and statistical analysis}

We developed a plug-in for the open source image processing software Image ${ }^{29}$ which was used to manually segment the vesicles from mosaics. Segmentation by an expert is the direct link between qualitative and quantitative data, and has to be executed with a high degree of confidence to allow reliable vesicle morphology detection with automatic image processing approaches. For every segmented vesicle, its properties (area, diameter, shape) were recorded.

\subsection{Hardware and Software Components}

All processing was performed on a PC with a Quad CPU at 2.33 GHz, 8 GB RAM, on Windows Server HPC 64-bit edition, 2007. The image processing algorithms were developed in MATLAB 2009b, the ImageJ plug-in "Shape Segmenter" was developed in Java with the use of the environment Eclipse. Microsoft Excel 2007 and MATLAB were used for statistical analysis. The invert microscope used was a Nikon Eclipse TE2000-S with an attached Sony CCD video camera module, model: XC-77 CE. 


\section{Results}

\subsection{Preprocessing of Video Sequences}

Video sequences, 768 pixels wide and 576 pixels high, were acquired at a rate of 25 frames per second and compressed with DivX video compression. Each video was then split into a sequence of individual frames, 1500 for every minute. The videos were recorded with a color camera, but since the colors contained no additional information, we converted all frames into grayscale-intensity values with equal regard to each of the three color channels $(\mathrm{RGB})[$ Intensity $=1 / 3 \times($ red + green + blue $)]$. All frames were de-interlaced with bicubic interpolation and every second de-interlaced frame was discarded. The frames had a thin black region on the sides and were thus cropped to a size of 762 $\times 570$ pixels.

Due to impurities in the microscope hardware (lenses, glasses, camera), some artifacts appeared on video sequence frames and had to be removed before proceeding (Fig. 4). To remove these artifacts, we selected a random subsample of $\sim 200$ frames of the pilot video sequence, and calculated the median intensity values of every pixel $\left(I_{\text {noise }}\right)$. This way, the median value of pixels which were not obstructed by lens noise resulted in the gray value of the background while the pixels representing lens noise appeared darker [Fig. 4(d)]. To remove this additive noise from the video sequence, each frame is filtered using:

$$
\begin{aligned}
I_{\text {clean }}(i, j) & =I_{\text {dirty }}(i, j)-\left[I_{\text {noise }}(i, j)-\operatorname{mean}_{i, j}\left(I_{\text {dirty }}\right)\right] \\
i & =1 \ldots M ; j=1 \ldots N,
\end{aligned}
$$

where $M$ and $N$ are the height and width of the frame and mean $_{i, j}(I)$ is the mean intensity value of the image $I$. This step was carried out for every frame of all video sequences using the noise image obtained from the pilot video sequence.

\subsection{Frame Registration}

The first step in mosaicing is to calculate the translation between frames of the video sequence. Each translation between two consecutive frames is presented as a vector with two values, pixel translation in vertical and horizontal directions. Even though sliding of the object glass during the recording in our experiments is supposed to be 1-dimensional, the cumulative
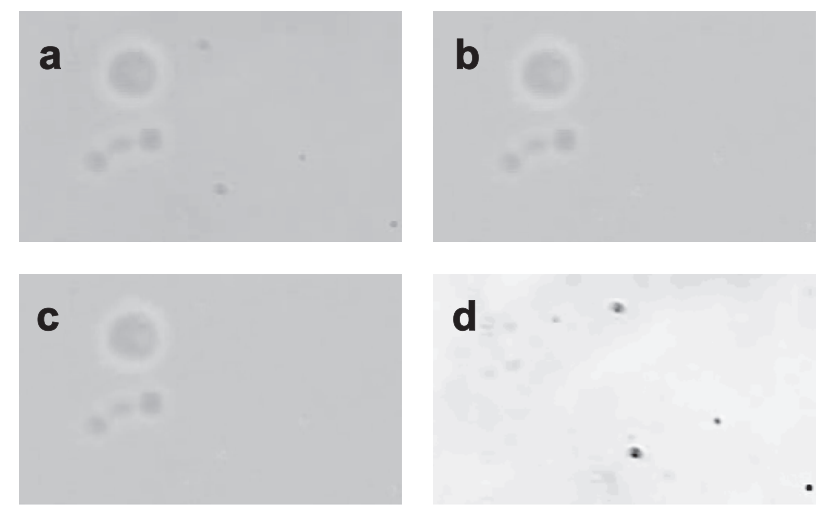

Fig. 4 Figures (a)-(d) show the same part of a video frame. (a) Original image, (b) zero median result after removal of artifacts, (c) after deinterlacing, and (d) additive noise artifacts. translation usually also reveals a small translation in the second dimension, due to the mechanical imprecision of the object glass slider. This is always smaller than $3 \%$ of the translation in the first dimension.

To calculate the translation, we find the peak value of the 2-dimensional normalized cross-correlation coefficient between the edge maps of each two consecutive frames. Proper filtering of the original images prior to edge estimation is a fundamental operation of image processing. A bilateral filter, which is an edge preserving smoothing technique, effectively a convolution with a non-linear Gaussian filter, with weights based on pixel intensities, is used. ${ }^{30}$ This results in blurring of generally flat surfaces such as the background, and consequent removal of the small glitches and undesired specimens out of the focal plane, without the loss of information on distinctive edges, in this case, the vesicle borders. Frames are then transformed into edge maps with the Sobel edge detector, ${ }^{31}$ using default settings in MATLAB 7.9.0, 2009b. We employ the 2-dimensional normalized cross correlation on these edge values instead of the intensity values when estimating the translation. The cumulative translations are then used to calculate the size of the mosaic - the image that represents the whole area recorded in the video sequence. When the translations from the first to the $i$ th frame are summed, the sum represents the exact location of the $i$ th frame inside the mosaic.

\subsection{Selecting the Best Frames for Mosaicing}

\subsubsection{Removing distorted frames}

Every vesicle was present in multiple consecutive frames, and the "frame quality," the sharpness of vesicles in the frames varied throughout the video sequence. When stitching a mosaic from a video sequence, there are many frames with an overlap of $90 \%$ or more pixels. It is crucial to discard the frames that hold imperfect, or skewed information or hold no new information at all. As the speed of object glass sliding was not uniform throughout the video, the moments when the object glass sliding was accelerated resulted in distorted frames. We designed a classifier to separate the sharp and useful frames from the distorted ones, which contained motion artifacts (Fig. 5). First, we randomly picked a subset of 500 frames (10\% of all frames) from the pilot video sequence and manually labelled them as "good" or "distorted", based on direct observation. These labelled frames were used to train a Linear Discriminant Analysis (LDA) classifier. ${ }^{32}$ When deciding which features to use for classification, multiple measures previously proposed for autofocusing in computer microscopy ${ }^{33}$ were compared. We calculated variance, contrast, entropy, Brenner gradient, ${ }^{34}$ and multiple image frequency based features for every frame of our video sequence. VizRank, ${ }^{35}$ a tool that automatically discovers and ranks interesting two-dimensional projections of class-labelled data, was employed to find the most promising features. Three features were selected. The first two were the Brenner gradient Eq. (2) and the contrast feature Eq. (3):

$$
\begin{aligned}
& \text { Brenner }=\sum_{i=1}^{N-2} \sum_{j=1}^{M}[I(i, j)-I(i+2, j)]^{2}, \\
& \text { Contrast }=\frac{\max _{i, j} I(i, j)-\min _{i, j} I(i, j)}{\max _{i, j} I(i, j)+\min _{i, j} I(i, j)},
\end{aligned}
$$



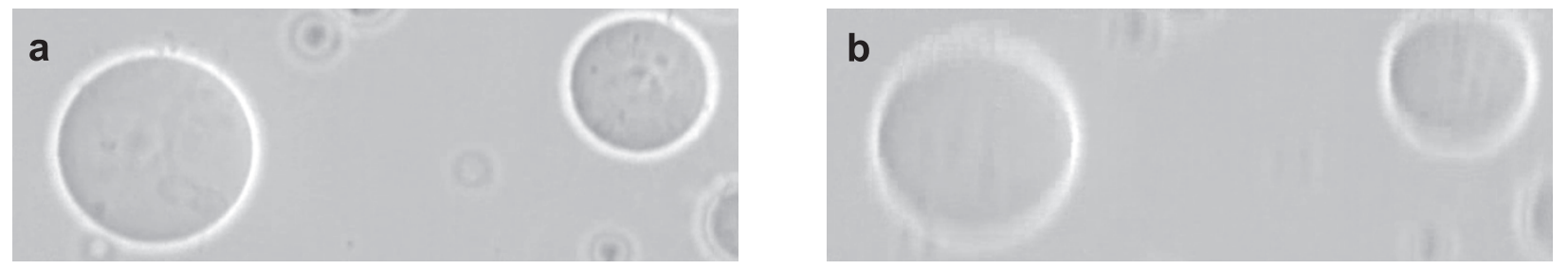

Fig. 5 (a) The vesicles have a sharp border. Frames containing sharp vesicles were labelled as "good" for the purpose of our classifier. (b) The frames, where the same vesicles are distorted due to a motion artifact which occurred when the movement of object glass under the microscope was accelerated. For the purpose of classification, these frames were labelled as "distorted".

where $M$ and $N$ are the height and width of the frame, and $I(i, j)$ is the intensity value. The third feature is based on the amplitude of the absolute frequency contained in the columns of the frame. For every frame, we compute the Absolute Frequency Amplitude $\left(\mathrm{AFA}_{\mathrm{s}}\right)$ which is the mean of the area under the frequency curve in the frequency bandwidth from $s$ to 1 over the columns of a single frame, where $0<\mathrm{s}<1$, corresponding to lowest and highest frequencies of the column respectively. $\mathrm{AFA}_{\mathrm{s}}$ is computed as follows:

$$
\mathrm{AFA}_{s}=\frac{1}{N} \sum_{f=s}^{M / 2} \sum_{j=1}^{N}\left|S_{j}(f)\right|,
$$

where $S_{j}(f)$ is the amplitude of the M-point Discrete Fourier Transform (DFT) frequency $f$ of the $j$ th column of the frame. The $\mathrm{AFA}_{\mathrm{s}}$ is then normalized by $\mathrm{AFA}_{0}$, the total absolute frequency amplitude under the frequency curve, which gives us the Absolute Frequency Amplitude Feature (AFAF). In other words, the AFAF is the ratio between the high pass that covers the top $67 \%$ of the frequency band, and the total absolute frequency amplitude under the frequency curve of the column:

$$
\mathrm{AFAF}=\frac{\mathrm{AFA}_{M / 6}}{\mathrm{AFA}_{0}} .
$$

The optimal $s$ numbers ( 0 and M/6) for AFAF in our classification were selected by random sampling, $\mathrm{M}$ in our case was 570 , which is the height of a frame column. This normalized metric (AFAF) is used as one of the three feature for classification.

Using these three features, the LDA classifier was used to separate the distorted and good frames. On a training set of 500 labelled frames, using cross-validation, LDA was on average able to correctly classify $90 \%$ of frames. This classifier, trained on 500 frames of the pilot video sequence was then successfully used to classify frames of the remaining video sequences. The LDA was used as the classifier because despite its simplicity, it provided a sufficiently accurate and generalized classification.

\subsubsection{Removing focusing frames}

Just as the sliding of the object glass was accelerated at some places, there was no sliding at all at some other places. This is most evident in the parts of the video sequence, where the operator stopped and adjusted the focal plane to find the sharpest representation of the vesicles in view. Due to the focus adjustments, the number of frames representing the same area during the adjustment accumulated by 25 every second. However, because of changing focus, the representation of vesicles in these frames varied from out of focus to in focus. For the mosaic stitching, we decided to keep only the frames where the vesicles are in focus. We introduced a new, "Focus measure," to compare subsequent frames for sharpness of vesicles (quality of focus) and select the sharpest ones. The training procedure to acquire the focus measure was conducted as follows. Six different focusing locations of the pilot video sequence presented as six highest peaks in graph (Fig. 6) were selected as data sets with 200 frames each. In all cases, less than 200 frames were necessary for the microscopist to adjust the frame focus. These six datasets (all together 1200 frames) were then used for training and testing of our Focus measure. These frames were manually labeled as the "good" frames (in focus) or "focusing" frames (to be discarded). Similarly to our "good" vs. "distorted" classification of frames (Sec. 3.3.1), multiple features were computed for every frame and VizRank was again employed to choose the optimal subset of features. The selected subset of features was composed of: the Brenner Gradient Eq. (2), the AFAF Eq. (5), and Entropy:

$$
\text { Entropy }=-\sum_{k=0}^{255} p(k) \cdot \log _{2} p(k),
$$

where $p(k)$ is the probability of $I(i, j)=k$ intensity. LDA was again employed to classify the "good" from the "focusing" frames, but this time the trained classifier was not used for classification. The output of the LDA is a discriminant hyperplane which best separates the two classes. Projecting the feature

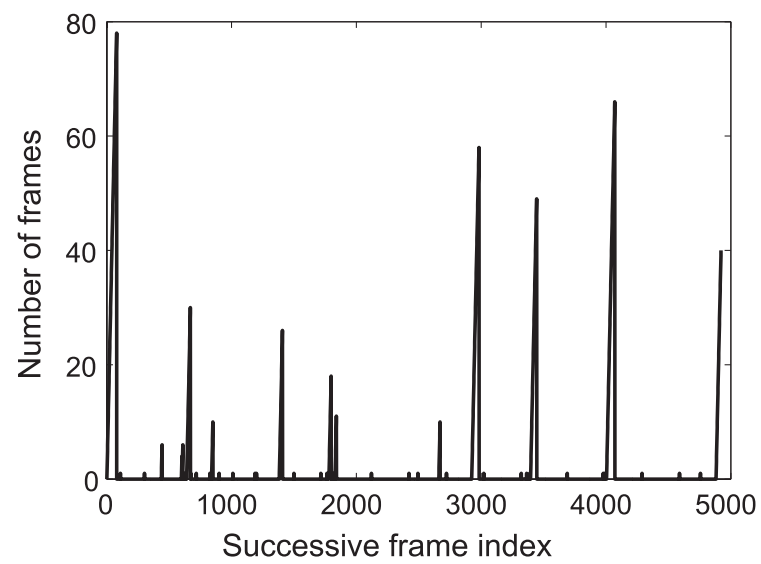

Fig. 6 The data in the graph is from a video sequence of 5,250 frames (3.5 min at 25 frames per second). Spikes in the graph present focusing locations where the spike height equals to the number of frames since last camera movement. The higher the spike, the more time (and consequently frames) was required for the operator to acquire a sharp image of the vesicles at that location. 

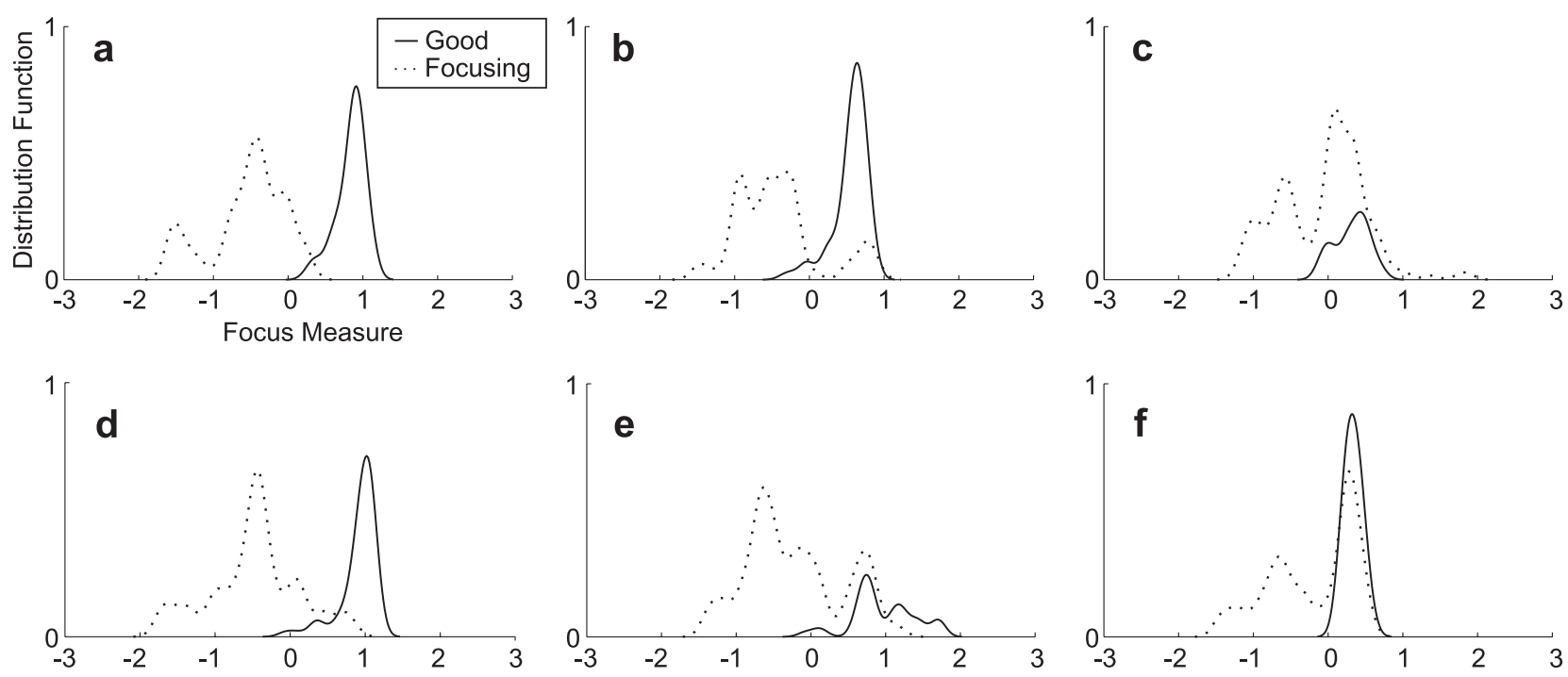

Fig. 7 Each of the six plots presents two density distributions of the focus measure values. The full line represents the distribution of values when calculated only for the frames labelled as good, and the dotted line represents the frames labelled as focusing. Six plots (a-f) represent six focusing locations of the pilot video sequence (six highest peaks of Fig. 6). In all six plots, the greater values of Focus measure correspond to frames in focus and the lower values correspond to frames out of focus.

vector of each micrograph onto the normal vector (vector inner product), which is perpendicular to the discriminant hyperplane, returns a scalar. In a classification problem, a threshold has to be set to allow separating the classes. Instead, projections of the micrograph feature vectors were used as a measure to compare frames for focus quality. As shown in Fig. 7, a greater focus measure value in a specific focusing situation can be associated with the frame which is generally more in focus. This focus measure is trained on the pilot video sequence and then additionally used to select the sharpest frames in the remaining video sequences. Wherever focus adjustments are encountered in a video sequence, the focus measure of all focusing frames is computed. Only the frames with the highest value are used for mosaic stitching.

\subsection{Buffered Stitching}

At the resolution of the video sequences in our experiment $(762 \times 570$ pixels $)$, the average non-zero vertical translation between consecutive frames was 9 pixels, which is approximately $1.5 \%$ of the frame height, and suggests a $98.5 \%$ overlap between successive frames. At a duration of $5 \mathrm{~min}$, which is the upper limit for our video sequence duration in this experiment, the video consists of roughly 7500 frames. Since our processing methods in Matlab require the image intensity values to be represented in a double format ( 8 bytes per pixel), the whole dataset requires $762 \times 570 \times 7500 \times 8 \mathrm{~B}=26 \mathrm{~GB}$ of RAM. In order to make our algorithms more general and applicable to different experiments and therefore longer video durations, we decided to break down the mosaic stitching into subsets of frames - buffers (Fig. 8). The accumulated memory constraint for each buffer can be limited to the available RAM in the computer used for mosaic stitching. Here, this constraint was set to limit the buffer size to 3 GB of RAM.

The vesicles in the video sequence are not completely uniformly distributed throughout the video, and some areas contain more vesicles than others. In order to find areas with fewer vesicles and use them for borders between buffers, we calculated the average variance of every horizontal line of the mosaic. The lines with the lowest LineVariance Eq. (7) were also the lines with few or no vesicles (Fig. 9). LineVariance for the $i$ th line is calculated as:

LineVariance $_{i}=\frac{1}{K \cdot N} \cdot \sum_{k=1}^{K} \sum_{j=1}^{N}\left(I_{k}(i, j)-\mu_{k}(i)\right)^{2}$,

where $N$ is the frame width, $I$ is the intensity, $i$ corresponds to the successive line in the mosaic, $\mu_{k}(i)$ is the mean intensity value of the $i$ th line in frame $k$ that contains this line in a given buffer, and $K$ is the number of frames that contain the $i$ th line. The process of separating the mosaic processing into manageable

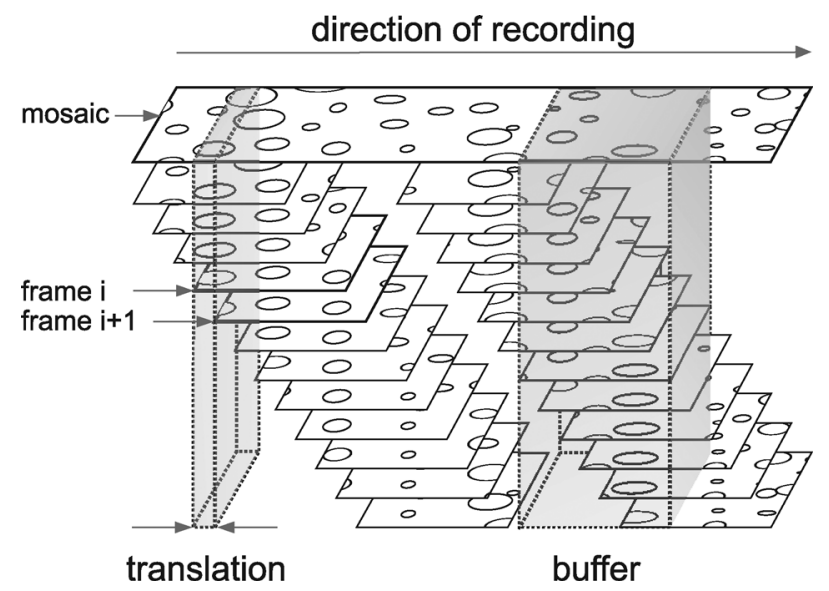

Fig. 8 Interpretation of frames of the video sequence in our algorithms. Translation, presented in the left shaded cubicle, is the movement between two consecutive frames. The right shaded cubicle represents a single buffer with all corresponding frames of the video sequence. Each buffer contained a full width slice of the mosaic. 


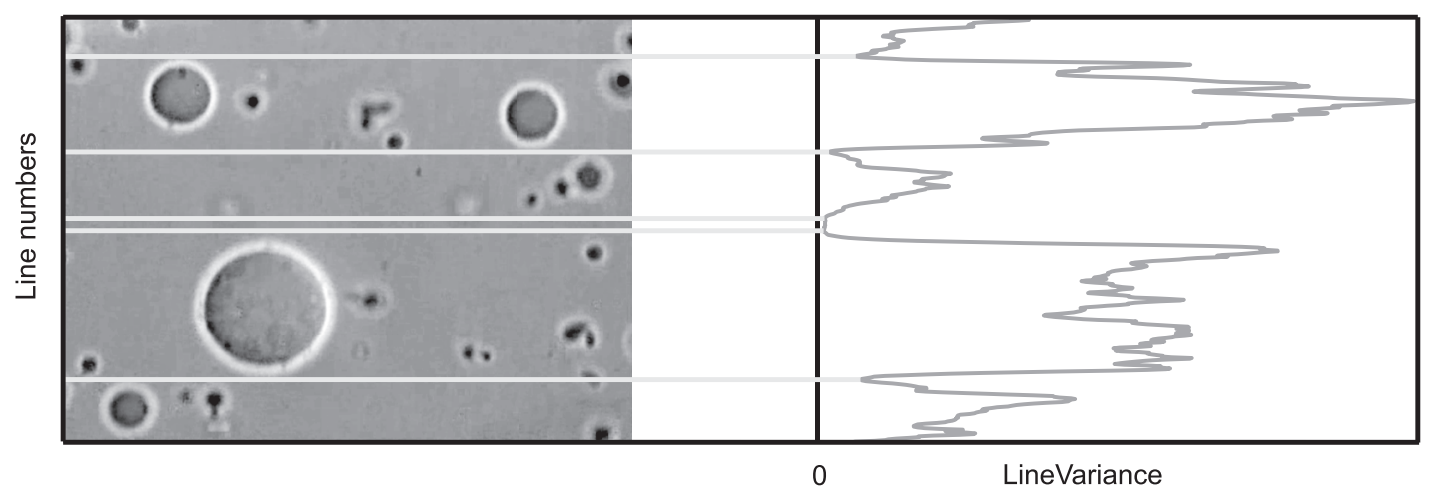

Fig. 9 Lines without vesicles in the frame on the left correspond to variance minima in the plot of LineVariance Eq. (7) on the right. Potential buffer borders are found in this way, and are marked by horizontal lines leading from the plot on the right to the frame on the left.

buffers was performed as a constrained optimization problem, as follows.

The function LineVariance, computed over the mosaic height was smoothed with a moving average of 20 and the local minima were extracted. We used a hierarchical approach to determine which of the local minima of LineVariance to use as buffer borders. This was done by sorting the local minima in ascending order and iteratively splitting bigger buffers of the mosaic into smaller and smaller buffers until every buffer size satisfied the preset memory constraint. To blend all frames of a single buffer into representation of the area, the median intensity value of frames included in the buffer is calculated. These median images of the buffers are then combined into the mosaic representing the entire area of the recorded video sequence (Fig. 8). The transitions between buffer borders are smooth and unnoticeable, because two consecutive buffers always contain (different) parts of the same frames. A part of a stitched mosaic is presented in Fig. 10.

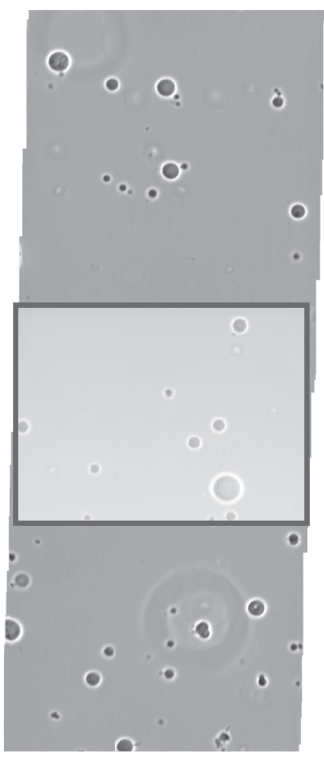

Fig. 10 Part of a mosaic stitched from a microscopy video sequence of a single track. The whole track is approximately 33 times the height of the presented sample. The rectangle in the centre represents a single field of view that can be observed at 400x magnification and is also the size of a single frame.

\subsection{Validation of Mosaic Stitching}

To validate the mosaic stitching, we inspected the mosaic and compared the vesicles in it with the vesicles in the original video sequence from which the mosaic was stitched. We found all vesicles in both, and were therefore able to confirm that the mosaics are valid representations of the video sequences. Additionally, the length of every mosaic in pixels was shown to be equal to the actual length of the recorded region in the specimen.

\subsection{Vesicle Segmentation and Statistical Analysis}

We developed a plug-in for open source software ImageJ that was used to manually segment the vesicles incubated in different media. Every vesicle was segmented with a unique colour representing the vesicle type (eight types all together), to form a mask (bottom row of Fig. 11) which was later extracted with MATLAB. The area of every vesicle was calculated, and for all spherical vesicles also their diameters. More than $90 \%$ of all detected vesicles were classified as spherical and the rest were nonspherical shapes.

\subsection{Vesicle Size and Shape Changes After Incubation}

This methodology was applied to three lipid vesicle populations to assess the shape and size transformations of the vesicles. The first population was exposed to neutral $\mathrm{CoFe}_{2} \mathrm{O}_{4}$ nanoparticles (CF), the second population to negatively charged citrate-coated $\mathrm{CoFe}_{2} \mathrm{O}_{4}$ nanoparticles (CF-CA), and the third population was left unexposed and used as a control $(\mathrm{C})$. The duration of exposure was $90 \mathrm{~min}$ in all three cases. We were able to detect the following differences between $\mathrm{CF}, \mathrm{CF}-\mathrm{CA}$, and $\mathrm{C}$ populations. The mean diameter of the spherical vesicles in the control population (C) decreased from $6.5 \mu \mathrm{m}$ to $6 \mu \mathrm{m}$ after 90 min incubation, while the mean diameters in the $\mathrm{CF}$ and CF-CA populations increased to $8.1 \mu \mathrm{m}$ and $8.5 \mu \mathrm{m}$, respectively [Fig. 12(a)]. Nonspherical vesicles were up to three times more likely to appear in $\mathrm{CF}$ and $\mathrm{CF}-\mathrm{CA}$ exposed populations than in the $\mathrm{C}$ population [Fig. 12(b)]. The differing occurrence of pearled vesicles was particularly noticeable [Fig. 12(c)]. Nonspherical vesicles represented from $5-15 \%$ of all vesicles in the exposed populations.

An additional detected difference was in the distribution of the diameter sizes of the vesicles in the populations. The results 

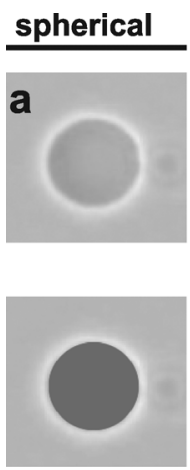
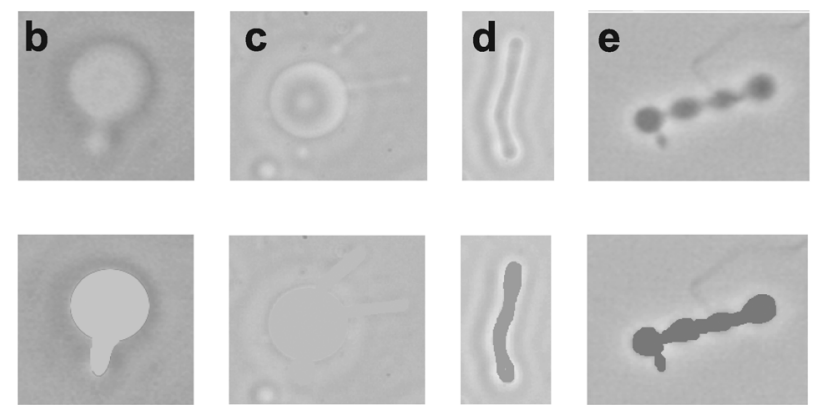

nonspherical
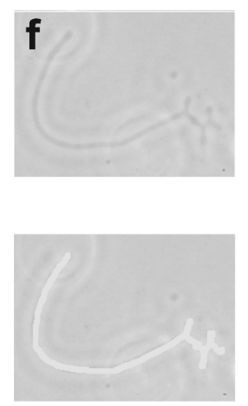

Fig. 11 Vesicles of all detected types manually labelled with our Shape Segmenter plug-in for ImageJ. The upper row shows the vesicle shapes and the lower row shows the way the vesicles in the upper row were segmented with the Shape segmenter ImageJ plug-in. (a) Spherical vesicle, (b-f) nonspherical vesicles, more specifically: (b) pear shaped vesicle, (c) circle with vesicles and tubes, (d) tube, (e) pearled vesicle, (f) long threaded pearled vesicle.

of the Kolmogorov-Smirnov test ${ }^{36}$ on the differences between the distributions are presented in Tab. 1 . We can observe minimal changes of cumulative distribution function (CDF) in the $\mathrm{C}$ population before and after $90 \mathrm{~min}$ of incubation, whereas results from the $\mathrm{CF}$ and $\mathrm{CF}-\mathrm{CA}$ populations suggest significantly more vesicles with larger diameter (Fig. 13). The total quantity of vesicles in all experimental populations together was 6,065 .

\section{Discussion}

The developed methodology enables extraction of data on size and shape transformations of thousands of POPC lipid vesicles incubated in different media. The protocol began with recording videos of vesicle populations exposed to different nanoparticles, then image processing methods were applied to form mosaics from the video sequences and finally, we segmented vesicles and conducted a statistical analysis of data extracted from the segmented vesicles. Using this approach, differences in the morphological properties of lipid vesicles incubated in a suspension with or without nanoparticles were demonstrated. In addition, differences in shape transformations of populations of lipid vesicles incubated with neutral cobalt-ferrite nanoparticles and those incubated with negatively charged citrate-coated cobalt-ferrite nanoparticles were detected.

Table 1 The diameter size distributions of spherical vesicles in populations were compared with a two-sample Kolmogorov-Smirnov test. The results of the statistical test on the distributions can be categorised as: $=$ equal, ${ }^{*} p<0.01,{ }^{* *} p<0.001,{ }^{* * *} p<0.0001$.

\begin{tabular}{cccccc} 
Duration $[\mathrm{min}]$ & 0 & \multicolumn{3}{c}{90} \\
\hline & Population & $\mathrm{C}$ & $\mathrm{C}$ & $\mathrm{CF}$ & CF-CA \\
0 & $\mathrm{C}$ & $=$ & $* *$ & $* * *$ & $* * *$ \\
90 & $\mathrm{C}$ & $* *$ & $=$ & $* * *$ & $* * *$ \\
& $\mathrm{CF}$ & $* * *$ & $* * *$ & $=$ & $* * *$ \\
& CF-CA & $* * *$ & $* * *$ & $* * *$ & $=$ \\
\hline
\end{tabular}

Our data on size and shape transformations of giant lipid vesicles incubated in a suspension of nanoparticles, confirm findings described by other authors. ${ }^{13,16}$ Incubation in a suspension of neutral $\mathrm{CoFe}_{2} \mathrm{O}_{4}(\mathrm{CF})$ or negatively charged citrate-coated $\mathrm{CoFe}_{2} \mathrm{O}_{4}$ nanoparticles (CF-CA), results in an increased quantity of nonspherical vesicles [Fig. 12(b)-12(c)], the increase being most notable in occurrences of pearled vesicles. ${ }^{16} \mathrm{Yu}$ et al. additionally reported formation of pearled vesicles as a result of interaction between lipid vesicles and negatively charged nanoparticles. They explained that cationic nanoparticles absorb onto vesicles causing a mismatch of surface area between the outer and inner leaflets of the bilayer result in pearl formation.

Studying the dynamic nature of shape transformations of vesicles under different stimuli is a challenging task $\mathrm{k}^{2,16,37}$ for which light microscopy is indispensable. However, when observed individually, only a limited number of vesicles can be investigated. By extending the light microscopy approach by means of providing data on large quantities of vesicles, more information on conformational behavior of vesicles can be extracted. The methodology presented and applied in our study offers this possibility. It enables the acquisition of video sequences of areas of the specimen, and stitching the video frames into a single large image, a mosaic. We succeeded in developing classifiers to remove frames of the video sequence that are out of focus and frames which are distorted due to motion artifacts which occur when the movement of object glass is accelerated. Image features such as the Brenner gradient, entropy, contrast and our Absolute Frequency Amplitude Feature are used for classification and reduction of video frames to those of better sharpness. Additionally, a hierarchical optimization algorithm is proposed to split the mosaic stitching into buffers. Thus, video sequences of arbitrary long duration can be stitched into mosaics on a computer with as little as 2 GB of RAM (minimal requirement recommendation).

Majority of the available commercial solutions for mosaicing focus on stitching together individual overlapping images only. However, when stitching frames of a video sequence, the large quantity of nearly completely overlapping frames makes these algorithms vastly memory intensive. Image registration in commercial software applications is generally computed between more than two consequent frames, which is incredibly 


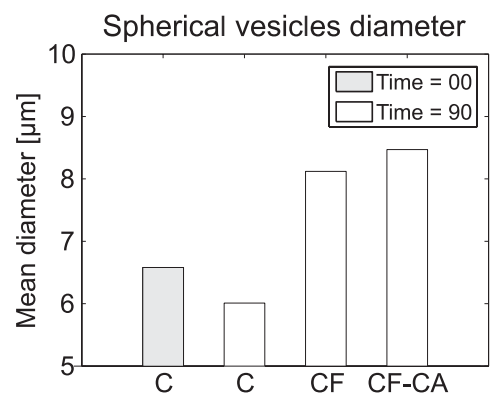

a

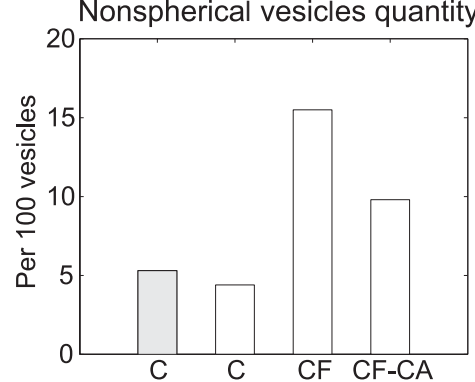

b

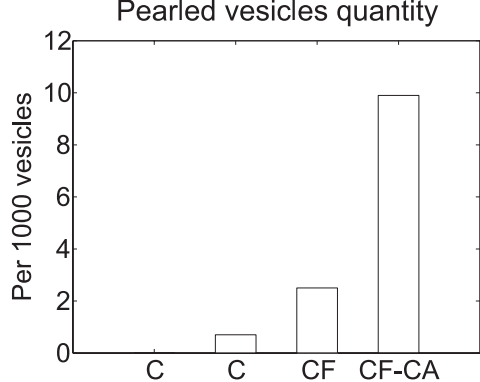

C

Fig. 12 (a) Mean diameter size of spherical vesicles for each experimental population, (b) number of nonspherical vesicles per 100 vesicles and (c) number of pearled vesicles per 1000 vesicles. The experimental populations are: C-control, CF-neutral Cobalt-ferrite nanoparticles and CF-CA-negatively charged citrate-coated Cobalt-ferrite nanoparticles. Vesicles in the C population were observed at time 0 , and vesicles in all three populations were observed after 90 min of incubation.

time consuming and can generate false translation information. Since all frames contain lipid vesicles, two frames, which are remotely separate in the video sequence, might be visually similar, and therefore falsely computed as having considerable overlap. We would like to mention that the proposed method is not optimal in any sense and we do not claim that it is superior to any other method. Our main purpose is to demonstrate that rapidly acquired lower quality frames in the form of a video, as opposed to a sequence of carefully acquired images with slower progression over the area, could be utilized for the analysis of nanoparticle effects on lipid vesicles. For this purpose, the proposed approach is novel, simple, and satisfactory.

In the future, the methodology can be improved by developing image segmentation algorithms which allow automatic segmentation of vesicles from our microscopy mosaics. This will permit completely automated processing of recorded video sequences and reduce the time required for vesicle segmentation

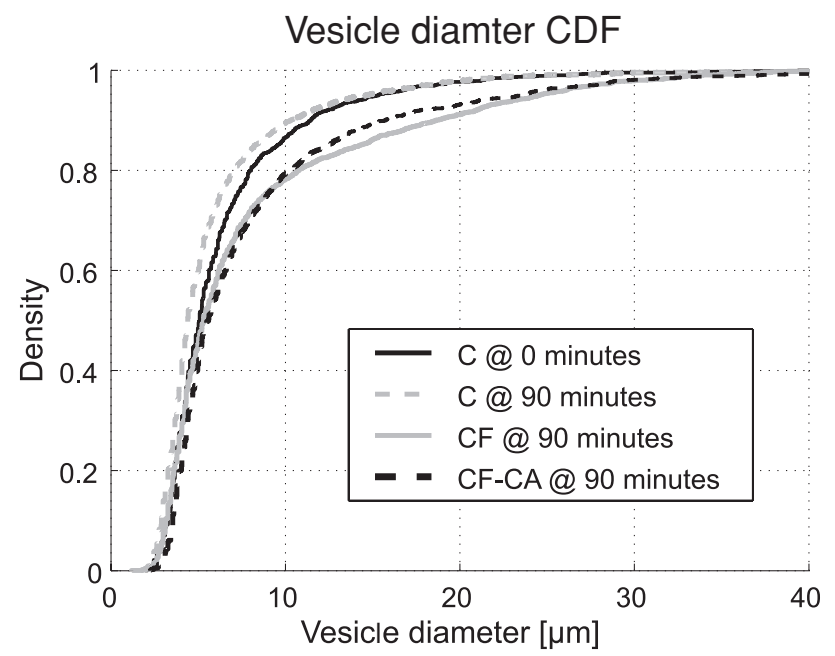

Fig. 13 Each curve presents a cumulative distribution function (CDF) of the diameters of spherical lipid vesicles. The $X$ axis gives sizes of the lipid vesicles in micrometers and $F(X)$ is percentage of all vesicles with diameter smaller than $X$. We can observe minimal changes of CDF in the $C$ group before and after 90 min of incubation. However, the distributions in the sizes of CF and CF-CA lipid vesicles suggest notably more vesicles with larger diameter. The vesicles with diameters above $40 \mu \mathrm{m}$ are not shown because vesicles they represent less than $0.5 \%$ of the vesicle population. from hours of input by experts, to minutes of computer processing. In addition, the repeatability of experiments will be tested and different time frames will be selected in order to allow us to follow a detailed time dependent response. The developed algorithms and plug-ins will be made available for other users to test shape transformations of lipid vesicles.

We conclude that the methodology presented holds much promise for future studies of responses of lipid vesicle populations to various substances. Among these, nanoparticles are perhaps the most challenging and interesting.

\section{Acknowledgments}

We would like to acknowledge the work of Sabina Boljte, Melita Korosec, Barbara Drasler, and contributions of Prof. Veronika Kralj-Iglic and Prof. Ales Iglic. The majority of the research conducted by Jernej Zupanc was funded by the Slovenian Research Agency, and part of his research visit at Northeastern University, USA, was funded by the U.S. Department of State as a Fulbright Scholarship. The work of Prof. Deniz Erdogmus and Erhan Bas was partially supported by NSF Grant Nos. ECS0524835/ECCS0929576 and ECS0622239/ECCS0934506. The work of Prof. Damjana Drobne was partially supported by two Centers of excellence (Advanced Materials and Technologies for the Future, Nanosciences and Nanotechnologies).

\section{References}

1. R. Lipowsky, "The Conformation of Membranes," Nature 349(6309), 475-481 (1991).

2. A. J. Markvoort, R. A. van Santen, and P. A. J. Hilbers, "Vesicle shapes from molecular dynamics simulations," J. Phys. Chem. B 110(45), 22780-22785 (2006).

3. F. M. Menger and M. I. Angelova, "Giant vesicles: Imitating the cytological processes of cell membranes," Acc. Chem. Res. 31(12), 789-797 (1998).

4. R. Dimova, S. Aranda, N. Bezlyepkina, V. Nikolov, K. A. Riske and R. Lipowsky, "A practical guide to giant vesicles. Probing the membrane nanoregime via optical microscopy," J. Phys.: Condens. Matter 18(28), S1151-S1176 (2006).

5. L. Mathivet, S. Cribier, and P. F. Devaux, "Shape change and physical properties of giant phospholipid vesicles prepared in the presence of an AC electric field," Biophys. J. 70(3), 1112-1121 (1996).

6. S. Svetina and B. Zeks, "Shape behavior of lipid vesicles as the basis of some cellular processes," Anat. Rec. 268(3), 215-225 (2002). 
7. P. Peterlin, G. Jaklic, and T. Pisanski, "Determining membrane permeability of giant phospholipid vesicles from a series of videomicroscopy images," Meas. Sci. Technol. 20(5) (2009).

8. L. R. Arriaga, R. Rodriguez-Garcia, I. López-Montero, B. Farago, T. Hellweg and F. Monroy, "Dissipative curvature fluctuations in bilayer vesicles: Coexistence of pure-bending and hybrid curvaturecompression modes," Eur. Phys. J. E 31(1), 105-113 (2010).

9. J. Pecreaux, H. G. Dobereiner, J. Prost, J.-F. Joanny, and P. Bassereau, "Refined contour analysis of giant unilamellar vesicles," Eur. Phys. J. E 13(3), 277-290 (2004).

10. T. Gruhn, T. Franke, R. Dimova, and and R. Lipowsky, "Novel method for measuring the adhesion energy of vesicles," Langmuir $\mathbf{2 3}(10), 5423$ 5429 (2007).

11. C. T. Leirer, B. Wunderlich, A. Wixforth, and M. F. Schneider, "Thermodynamic relaxation drives expulsion in giant unilamellar vesicles," Phys. Biol. 6(1), 4 (2009).

12. J. Barauskas, C. Cervin, M. Jankunec, M. Špandyreva, K. Ribokaite, F. Tibergb and M. Johnssonb, "Interactions of lipid-based liquid crystalline nanoparticles with model and cell membranes," Int. J. Pharm. 391(1-2), 284-291 (2010).

13. B. Wang, L. F. Zhang, S. C. Bae, and S. Granick, "Nanoparticle-induced surface reconstruction of phospholipid membranes," Proc. Natl. Acad. Sci. U.S.A. 105(47), 18171-18175 (2008).

14. R. Lipowsky and H. G. Dobereiner, "Vesicles in contact with nanoparticles and colloids," Europhys. Lett. 43(2), 219-225 (1998).

15. P. R. Leroueil, S. Y. Hong, A. Mecke, J. R. Baker, Jr, B. G. Orr, and M. M. Banaszak Holl, "Nanoparticle interaction with biological membranes: Does nanotechnology present a janus face?," Acc. Chem. Res. 40(5), 335-342 (2007).

16. Y. Yu and S. Granick, "Pearling of Lipid Vesicles Induced by Nanoparticles," J. Am. Chem. Soc. 131(40), 14158-14159 (2009).

17. J. Zupanc, J. Valant, D. Drobne, V. K. Iglič, and A. Iglič, "A new approach to analyse effects of nanoparticles on lipid vesicles," Int. J. Biomed. Nanosci. Nanotechnol. 1(1), 34-51 (2010).

18. S. Bae, S. W. Lee, Y. Takemura, Y. H. Jo, S. G. Lee, "AC MagneticField-Induced Heating and Physical Properties of Ferrite Nanoparticles for a Hyperthermia Agent in Medicine," IEEE Trans. Nanotechnol. 8(1), 86-94 (2009).

19. B. Ma, T. Zimmermann, M. Rohde, S. Winkelbach, F. He, W. Lindenmaier, and K. E. J. Dittmar, "Use of Autostitch for automatic stitching of microscope images," Micron 38(5), 492-499 (2007).

20. A. Campilho and M. Kamel, "A Stitching Algorithm for Automatic Registration of Digital Radiographs," Lect. Notes Comput. Sci.: Image Analysis and Recognition 5112, 854-862 (2008).

21. W. Mitzner, J. Fallica, and J. Bishai, "Anisotropic Nature of Mouse Lung Parenchyma," Ann. Biomed. Eng. 36(12), 2111-2120 (2008).
22. D. S. Gareau, Y. B. Li, K. S. Nehal, M. Rajadhyaksha, B. Huang, and Z. Eastman, "Confocal mosaicing microscopy in Mohs skin excisions: feasibility of rapid surgical pathology," J. Biomed. Opt. 13(5) 054001-12 (2008).

23. J. Kopf, M. Uyttendaele, O. Deussen, and M. F. Cohen, "Capturing and viewing Gigapixel images," ACM Trans. Graphics 26(3), 93-102 (2007).

24. S. K. Chow, H. Hakozaki, D. L. Price, N. A. B. Maclean, T. J. Deerinck, J. C. Bouwer, M. E. Martone, S. T. Peltier and M. H. Ellisman, "Automated microscopy system for mosaic acquisition and processing," J. Microsc.-Oxford 222, 76-84 (2006).

25. S. De Backer, F. Cornelissen, J. Lemeire, R. Nuydens, T. Meert, P. Schelkens, and P. Schelkens, "Mosaicing of Fibered Fluorescence Microscopy Video," in Advanced Concepts for Intelligent Vision Systems, Lect. Notes Comput. Sci. 5259, 915-923 (2008).

26. M. I. Angelova, S. Soléau, P. Meleard, F. Faucon, and P. Bothorel, "Preparation of giant vesicles by external AC electric fields. Kinetics and applications," Trends Colloid Interface Sci. 89, 127-131 (1992).

27. V. Kralj-Iglic, G. Gomiscek, J. Majhenc, V. Arrigler, and S. Svetina, "Myelin-like protrusions of giant phospholipid vesicles prepared by electroformation," Colloids Surf. A 181(1-3), 315-318 (2001).

28. H. G. Dobereiner, E. Evans, M. Kraus, U. Seifert, and M. Wortis, "Mapping vesicle shapes into the phase diagram: A comparison of experiment and theory," Phys. Rev. E 55(4), 4458-4474 (1997).

29. M. D. Abramoff, P.J. Magelhaes, and S.J. Ram, "Image Processing with ImageJ,” Biophotonics Int. 11(7), 36-42, (2004).

30. C. Tomasi and R. Manduchi, "Bilateral Filtering of Gray and Colored Images." in Proc. IEEE International Conference on Computer Vision (1998).

31. R. C. Gonzales and R.C. Woods, "Digital image processing," AddisonWesley, Reading, MA (1992).

32. G. J. McLachlan, "Discriminant Analysis and Statistical Pattern Recognition," Wiley Interscience (2004).

33. Y. Sun, S. Duthaler, and B. J. Nelson, "Autofocusing in computer microscopy: Selecting the optimal focus algorithm," Microsc. Res. Tech. 65(3), 139-149 (2004).

34. S. Yazdanfar, K. B. Kenny, K. Tasimi, A. D. Corwin, E. L. Dixon, and R. J. Filkins, "Simple and robust image-based autofocusing for digital microscopy," Opt. Express 16(12), 8670-8677 (2008).

35. G. Leban, I. Bratko, Uros Petrovic, T. Curk and B. Zupan, "VizRank: finding informative data projections in functional genomics by machine learning," Bioinformatics 21(3), 413-414 (2005).

36. F. J. Massey, "The Kolmogorov-Smirnov test for goodness of fit," J. Am. Stat. Assoc. 46(253), 68-78 (1951).

37. J. Kas and E. Sackmann, "Shape transitions and shape stability of giant phospholipid-vesicles in pure water induced by area-to-volume changes," Biophys. J. 60(4), 825-844 (1991) . 